\title{
Perfil neuropsicológico de adultos com queixas de desatenção: diferenças entre portadores de TDAH e controles clínicos
}

\section{Neuropsychological profile of adults with attention deficits: differences between ADHD and clinical controls}

\author{
Cíntia Mesquita ${ }^{1}$, Gabriel Coutinho², Paulo Mattos 3 \\ - Psicóloga do Centro de Neuropsicologia Aplicada (CNA) e pesquisadora do Grupo de Estudos do Déficit de Atenção e Hiperatividade (GEDA) do Instituto de Psiquiatria da Universidade Federal do \\ Rio de Janeiro (UFRJ). \\ ${ }_{2}$ Psicólogo do CNA, membro dos Laboratórios Integrados de Neuropsicologia (Lineu). \\ ${ }_{3}$ Psiquiatra, coordenador do GEDA, diretor clínico do CNA, membro dos Lineu.
}

Recebido: 25/6/2009 - Aceito: 11/11/2009

\begin{abstract}
Resumo
Contexto: A distinção entre portadores e não portadores de transtorno do déficit de atenção e hiperatividade (TDAH) com queixas de desatenção representa um desafio clínico frequente, aspecto agravado pelo predomínio de estudos na literatura que comparam os perfis de portadores a controles normais, mas não a controles clínicos com queixas atencionais. Objetivo: Comparar o desempenho neuropsicológico de adultos portadores de TDAH segundo o Manual de Diagnóstico e Estatística das Perturbações Mentais (DSM-IV) com controles clínicos com queixas de desatenção encaminhados para avaliação neuropsicológica. Métodos: Foram obtidos retrospectivamente prontuários relativos a todos os indivíduos adultos que apresentavam desatenção como queixa principal, encaminhados para avaliação neuropsicológica num intervalo de dois anos em serviço especializado, e comparados todos os portadores de TDAH ( $\mathrm{n}=56$; 64,28\% de homens) a uma amostra de controles clínicos extraídos da mesma amostra, sem TDAH ( $\mathrm{n}=29 ; 58,62 \%$ de homens), com idades entre 18 e 59 anos. Foram utilizadas medidas de atenção, funções executivas e memória. Resultados: Os grupos revelaram-se pareados quanto a idade, escolaridade, distribuição por sexo e QI ( $p>0,05$ ). Não foram observadas diferenças entre os grupos nos testes formais para avaliação de capacidade atencional; entretanto, o grupo de portadores de TDAH apresentou pior desempenho em tarefas que envolviam memória operacional auditivo-verbal. Conclusão: Adultos portadores de TDAH podem se diferenciar de controles clínicos encaminhados com queixas de desatenção pelo desempenho deficitário em tarefas que endereçam memória operacional auditivo-verbal.
\end{abstract}

Mesquita C, et al. / Rev Psiq Clín. 2010;37(5):212-5

Palavras-chave: Transtorno do déficit de atenção e hiperatividade, TDAH, testes neuropsicológicos.

\begin{abstract}
Background: Discriminating attention deficit hyperactivity disorder (ADHD) from non-ADHD individuals referred to evaluate attention deficits is a challenge that is often faced by clinicians, which might be aggravated by the design of the majority of studies that compare ADHD to normal control comparison individuals, instead of comparing to control clinical individuals. Objective: To compare neuropsychological performance of DSM-IV ADHD adults and clinical control individuals with attention complaints referred for neuropsychological evaluation. Methods: We conducted a retrospective analysis through the database of a private clinic specialized in neuropsychological evaluation along a two year period. ADHD adults ( $\mathrm{n}=56 ; 64.28 \%$ male) were compared to control individuals from the same sample referred due to inattention ( $n=29 ; 58.62 \%$ male), with an age range from 18 to 59 years old. Measures of attention, executive functions and memory were used. Results: Groups were paired by age, schooling level, gender distribution and IQ ( $p>0.05)$. We found no differences regarding performances in attention tasks; however, ADHD individuals showed worse performances in tasks that demand verbal working memory. Discussion: When comparing ADHD adults and control individuals with complaints of attention deficits, the first group presented worse performance only in verbal working memory tasks.
\end{abstract}

Mesquita C, et al. / Rev Psiq Clín. 2010;37(5):212-5

Keywords: Attention deficit hyperactivity disorder, ADHD, neuropsychological tests.

\section{Introdução}

A forma adulta do transtorno do déficit de atenção e hiperatividade (TDAH) é oficialmente reconhecida e amplamente estudada, tendo uma prevalência estimada em torno de $4 \%{ }^{1}$. Estudos brasileiros com adultos portadores demonstraram que a forma adulta do TDAH frequentemente se associa a altas taxas de comorbidades ${ }^{2,3}$, o que não apenas agrava o quadro sintomático, como representa um desafio clínico, uma vez que diversos transtornos neuropsiquiátricos podem apresentar quadro sintomático semelhante ao do TDAH. Mais ainda, a distinção entre adultos portadores de TDAH e não portadores com queixas de desatenção pode ser particularmente difícil, em especial pela pobreza de estudos comparando esses dois grupos: a literatura apresenta quase invariavelmente estudos comparando portadores a controles normais, o que não reproduz a prática clínica. Outra dificuldade para estabelecimento de diagnóstico de TDAH deve-se à necessidade de haver múltiplos informantes, uma vez que diferentes fontes de informações podem apresentar baixa concordância, tanto para crianças (pais e professores) ${ }^{4}$ quanto para adultos (autorrelato comparado a informantes colaterais, tais como cônjuges ou outros familiares) 5 .

O estudo da Universidade de Massachusetts (the UMASS study) explorou as diferenças entre discriminação de portadores de TDAH e controles provenientes de comunidade e controles provenientes de amostras clínicas referidas. Os achados demonstraram que a utilização de apenas um único sintoma do módulo de desatenção poderia discriminar corretamente portadores de TDAH e controles de amostra não clínica com $97 \%$ de precisão. Por outro lado, a utilização de três itens de desatenção permitia diferenciar portadores de controles clínicos referidos com precisão de $87 \%$, demonstrando que indivíduos sem queixas são, de fato, mais facilmente discriminados 
de portadores, embora esses controles não façam parte do dia a dia clínico exatamente pelo fato de não apresentarem queixas.

O perfil neuropsicológico de adultos portadores de TDAH é semelhante àquele encontrado em crianças, apesar de cursarem com expressão fenotípica algo diferente, por conta dos contextos diversos que ocorrem nessas faixas etárias, tais como maiores demandas sobre funções executivas ${ }^{7}$. Uma das teorias mais consolidadas para explicar as manifestações neuropsicológicas do TDAH foi postulada por Barkley ${ }^{8}$, que propunha que os sintomas estariam associados primordialmente a um déficit de controle inibitório, sugerindo que o TDAH fosse um transtorno que cursa com déficits de funções executivas (FEs). Uma série de estudos posteriores demonstrou que, embora déficits de FEs fossem frequentemente demonstrados em portadores de TDAH, estes não seriam suficientes nem necessários para predizer todos os casos do transtorno ${ }^{9}$. Outros estudos demonstraram que portadores que apresentam déficits de FEs avaliados por testes neuropsicológicos apresentavam também maior comprometimento funcional que aqueles portadores que não apresentavam tais déficits ${ }^{10}$. Outras teorias que buscavam explicar os déficits neuropsicológicos no TDAH, dentre elas a Aversão ao Adiamento"11, a Regulação do Estado $^{12}$ e o Déficit de Transferência Dopaminérgica ${ }^{13}$, foram propostas por diferentes autores.

Com base em análise fatorial de baterias neuropsicológicas, alguns autores sugeriram que tarefas de FEs compreendem ao menos quatro fatores: controle inibitório e execução, memória operacional, alternância de conceitos e controle de interferência ${ }^{9}$. A memória operacional é um componente das FEs que permite que informações sejam armazenadas e monitoradas temporariamente ${ }^{14}$, sendo de fundamental importância para execução de diversas atividades mentais, como resolução de problemas, tomada de decisão, cálculos mentais e leitura ${ }^{15}$. O referido modelo pode ser entendido como um modelo híbrido, uma vez que conta com diferentes componentes, como executivo central (EC), alça fonológica, rascunho visoespacial e retentor episódico. Brevemente, o primeiro dos componentes seria responsável pelo controle do processamento, enquanto a alça fonológica e o rascunho visoespacial fariam armazenamento de informações (fonológico e visual, respectivamente); o retentor episódico seria responsável pela integração dos diferentes tipos de informação (fonológico, visual e provenientes de memória de longo prazo) ${ }^{14}$.

Alguns estudos realizados em nosso meio compararam desempenho de crianças com TDAH e controles em testes neuropsicológicos (por exemplo, Coutinho et al. ${ }^{16}$ ). No entanto, poucos estudos brasileiros avaliaram o perfil neuropsicológico de adultos com TDAH; também se observa escassez de testes neuropsicológicos validados para utilização em nosso meio, principalmente para adultos. Recentemente, foram apresentados a validação e o poder discriminativo do Iowa Gambling Task (IGT), um teste computadorizado que objetiva avaliar a capacidade decisória e a impulsividade cognitiva ${ }^{17}$. Esforços têm sido realizados visando adaptar novos instrumentos, como a bateria de inteligência para adultos (WAIS-III) e a tarefa de lista de palavras ${ }^{18}$. Embora testes neuropsicológicos não sejam suficientes nem necessários para o diagnóstico de $\mathrm{TDAH}^{19}$, estes podem ser úteis para consolidação do diagnóstico por meio da obtenção de dados objetivos ${ }^{20}$.

Um dos poucos estudos brasileiros com adultos portadores de TDAH demonstrou que eles apresentavam maior impulsividade tanto em testes neuropsicológicos quanto em escalas autopreenchidas ${ }^{21}$. O mesmo estudo demonstrou haver correlação entre impulsividade autorrelatada pela Escala BIS 22 e testes neuropsicológicos, sugerindo que ambos avaliam construtos semelhantes.

O objetivo do presente estudo foi comparar o desempenho neuropsicológico de adultos portadores de TDAH e controles de uma amostra clínica, empregando alguns dos testes mais utilizados em nosso meio. Esse mesmo desenho - isto é, comparação de portadores de TDAH e controles com queixas de desatenção - foi utilizado recentemente em outro estudo realizado por nosso grupo ${ }^{23}$. Neste estudo, crianças portadoras de TDAH foram comparadas a crianças sem esse diagnóstico e, embora em ambos os grupos houvesse queixas de mau desempenho acadêmico, o grupo com diagnóstico de TDAH apresentou desempenho inferior a controles em tarefas de sustentação da atenção e memória operacional auditivo-verbal. Com base nos achados da literatura, nossa hipótese foi de que portadores apresentariam desempenho levemente inferior ao de controles, principalmente em tarefas de atenção e memória operacional auditivoverbal. O estudo foi aprovado pelo Conselho de Ética em Pesquisa do Instituto de Psiquiatria da UFRJ (IPUB).

\section{Métodos}

Foi realizada uma análise retrospectiva de banco de dados de serviço privado especializado em avaliação neuropsicológica (Centro de Neuropsicologia Aplicada). Adultos com idades entre 18 e 59 anos foram encaminhados para avaliação num intervalo de dois anos, com queixas de desatenção. Foram identificados todos os indivíduos que apresentaram diagnóstico final de TDAH, de acordo com os critérios do sistema DSM-IV $(\mathrm{N}=88)$. Foram excluídos pacientes $(\mathrm{N}=$ 32) que apresentassem comorbidade atual (mas não pretérita) com transtornos de humor e de ansiedade $(\mathrm{N}=22)$; foram excluídos os pacientes com comorbidade de vida inteira (lifetime) com transtornos por uso de substâncias psicoativas e álcool $(\mathrm{N}=9)$, transtornos da aprendizagem $(\mathrm{N}=5)$, epilepsia $(\mathrm{N}=3)$ e transtornos de personalidade $(\mathrm{N}=1)$. Também foram excluídos todos os indivíduos com QI inferior a $80(\mathrm{~N}=4)$. Alguns pacientes apresentavam mais de um desses diagnósticos. Utilizou-se a entrevista semiestruturada Mini-Plus ${ }^{24}$ para investigar a presença dos principais transtornos do Eixo-I. Indivíduos também foram entrevistados utilizando-se instrumento baseado em critérios do DSM-IV para avaliar TDAH, além de preencherem a escala ASRS 25 e serem submetidos à entrevista clínica com psiquiatra. Os diagnósticos eram confirmados após reunião coordenada por psiquiatra sênior com título de especialista (PM). Nenhum dos indivíduos havia feito tratamento prévio para TDAH; a quase totalidade dos indivíduos havia sido encaminhada para avaliação pelo médico-assistente.

O grupo de controle foi composto por indivíduos que procuraram atendimento para avaliação de dificuldades atencionais e/ ou suspeita de TDAH, mas que não receberam esse diagnóstico ao final, tendo sido submetidos aos mesmos procedimentos do grupo experimental.

Foram utilizados os seguintes testes neuropsicológicos: Span de Dígitos (bateria WAIS-III) como medida de memória operacional auditivo-verbal, tanto alça fonológica (Span Forward) quanto EC (Span Backward); subteste Aritmética da bateria WAIS-III, por ser uma medida indireta de memória operacional; Stroop C/CW como medida de controle inibitório; Figura Complexa de Rey como medida de memória visual; Trail Making A e B como medidas de destreza visomotora (trilhas A e B) e flexibilidade cognitiva (trilhas B).

\section{Análise estatística}

Utilizou-se teste não paramétrico (teste U de Mann-Whitney) para comparar os grupos experimental (TDAH) e controle nas variáveis que não apresentaram distribuição normal (idade e escolaridade); foi utilizado teste paramétrico ( $\mathrm{t}$ de Student) para comparar os grupos naquelas variáveis que apresentaram distribuição normal (variáveis neuropsicológicas). Utilizou-se o teste de qui-quadrado para comparar distribuição por gênero entre os grupos.

\section{Resultados}

Cinquenta e seis portadores de TDAH foram incluídos no estudo. Observou-se predomínio de homens $(64,28 \%)$ no grupo TDAH. O grupo de controle foi composto por 29 indivíduos, sendo a maioria deles do sexo masculino (58,62\%). Não se observaram diferenças entre os grupos quanto à distribuição por gênero $(\mathrm{p}=0,609)$. A idade média do grupo de portadores foi de 29,00 anos $(D P=9,61)$, ao passo que a idade média de indivíduos do grupo de controle foi de 29.07 (DP = 7,58). O QI médio do grupo de TDAH foi de 97,38 (DP $=8,97)$, ao passo que o do grupo de controle foi de 95,97 $(\mathrm{DP}=9,20)$. Portadores de TDAH e controles estavam pareados quanto a idade $(\mathrm{p}=0,574)$, escolaridade $(\mathrm{p}=0,745)$ e QI $(\mathrm{p}=0,498)$ (Tabela 1$)$. 
Tabela 1. Características da amostra

\begin{tabular}{l|c|c|c}
\hline \multirow{2}{*}{ Medida } & \multicolumn{2}{|c|}{ Grupo } & \multirow{2}{*}{ Valor de $\mathrm{p}$} \\
\cline { 2 - 3 } & Controle $(\mathrm{n}=29)$ & TDAH $(\mathrm{n}=56)$ & \\
\hline Idade & 29,07 & 29,00 & 0,574 \\
\hline Escolaridade & 13,38 & 13,75 & 0,745 \\
\hline QI & 95,97 & 97,38 & 0,498 \\
\hline
\end{tabular}

Obs.: Comparações foram realizadas utilizando o teste U de Mann-Whitney.

\section{Variáveis neuropsicológicas}

O grupo de portadores de TDAH apresentou pior desempenho que os controles apenas em tarefas que envolviam memória operacional auditivo-verbal (Span de Dígitos), tanto na ordem direta (Span Forward) quanto na ordem inversa (Span Backward) ( $\mathrm{p}=0,009$ e $\mathrm{p}$ $=0,001$, respectivamente). Não foram observadas diferenças estatisticamente significativas nas outras variáveis neuropsicológicas. Os resultados estão descritos na tabela 2 .

Tabela 2. Desempenho nos testes neuropsicológicos

\begin{tabular}{l|c|c|c}
\hline \multirow{2}{*}{ Medida } & \multicolumn{2}{|c|}{ Grupo } & \multirow{2}{*}{ Valor de $\mathrm{p}$} \\
\cline { 2 - 3 } & Controle $(\mathrm{n}=29)$ & TDAH $(\mathrm{n}=56)$ & \\
\hline Span Forward & 9,55 & 6,68 & $0,009^{*}$ \\
\hline Span Backward & 6,48 & 4,93 & $0,001^{*}$ \\
\hline Aritmética & 11,34 & 11,59 & 0,756 \\
\hline Trilhas A & 34,35 & 36,59 & 0,605 \\
\hline Trilhas B & 58,65 & 70,38 & 0,086 \\
\hline Rey cópia & 33,89 & 35,16 & 0,180 \\
\hline Rey recuperação & 24,43 & 23,68 & 0,653 \\
\hline Stroop C & 112,00 & 109,76 & 0,458 \\
\hline Stroop CW & 103,09 & 96,08 & 0,194 \\
\hline
\end{tabular}

Obs.: Comparações foram realizadas utilizando teste $t$ de Student. * Controle $>$ TDAH.

\section{Discussão}

O desenho utilizado neste estudo pretendeu representar as dificuldades vivenciadas pelo clínico no cotidiano, isto é, discriminar indivíduos portadores de TDAH dentre o universo de indivíduos referindo queixas de desatenção. A ausência de diferenças estatisticamente significativas entre os grupos TDAH e controle nas variáveis sociodemográficas - distribuição por gênero, idade e escolaridade -, bem como QI, indica não haver evidências de que nenhuma dessas variáveis possa ter influenciado os outros resultados.

No presente estudo, observou-se predomínio do sexo masculino, contrariando achados de outros estudos de nosso meio que também utilizaram amostras clínicas de adultos ${ }^{3,26}$; não foi possível, entretanto, identificar possíveis razões para isso.

Portadores de TDAH apresentaram desempenho significativamente inferior aos controles em tarefa de memória operacional auditivo-verbal (Span de Dígitos), tanto na ordem direta quanto inversa, o que corrobora achados de alguns estudos com amostras de adultos e de crianças 23,27 .

Não foram observadas diferenças significativas entre os grupos nas outras variáveis neuropsicológicas investigadas. Alguns autores sugeriram que a ausência de discrepância entre portadores e não portadores de TDAH em testes neuropsicológicos pode ser um resultado esperado, tendo em vista que grande parte dos testes para FEs não foi desenvolvida para avaliação de déficits mais sutis, como aqueles observados em portadores de TDAH ${ }^{28}$. Biederman et al. ${ }^{29}$ demonstraram também que resultados comprometidos em testes de FEs refletiam mais frequentemente índices de QI mais baixos que comprometimento de FEs em atividades de vida diária per se.

Na mesma linha, McGough e Barkley ${ }^{19}$ sugeriram que, até o momento, testes de FEs não demonstraram valores preditivos positivo ou negativo que justificassem seu uso na prática clínica. Estudos brasileiros com adultos portadores de TDAH demonstraram que testes mais sensíveis para FEs podem ser capazes de discriminar grupos, além de ressaltarem a importância da utilização de questionários autopreenchidos ${ }^{21}$. Deve-se também ressaltar que nosso grupo de controle difere do habitualmente utilizado em outros estudos, tendo em vista que, embora os participantes não tenham diagnósticos psiquiátricos, eles vieram ao nosso serviço visando à avaliação neuropsicológica, ao contrário dos controles sem queixas utilizados em outros estudos.

Nossos achados devem ser entendidos à luz de algumas limitações: 1) nossa amostra é de tamanho reduzido, o que pode ter diminuído o poder estatístico, dificultando demonstração de discrepâncias entre desempenho dos grupos de controle e experimental; 2) utilizou-se amostra clínica de alto nível socioeconômico, o que dificulta a generalização de nossos resultados; 3 ) não se incluíram alguns dos testes considerados mais sensíveis para TDAH, como o IGT e o CPT-II, o que deve ser considerado como uma importante limitação.

\section{Conclusão}

Nossos achados indicam que as principais diferenças observadas entre portadores adultos de TDAH e controles clínicos que procuraram avaliação especializada encontram-se em tarefas que envolvem aspectos auditivo-verbais de memória operacional. Não foram observadas discrepâncias em outros testes para avaliação de FEs. Vale ressaltar que, apesar das diferenças encontradas no desempenho dos grupos de controle e TDAH nos testes, testes neuropsicológicos não são suficientes nem necessários para definir o diagnóstico de indivíduos que procuram atendimento. Entretanto, o exame neuropsicológico, ao ser interpretado pelo clínico, pode ser importante em algumas questões, tais como ${ }^{20}: 1$ ) prestar auxílio para consolidar ou excluir o diagnóstico de TDAH; 2) auxiliar no diagnóstico diferencial com outros transtornos; 3) auxiliar no estabelecimento de comorbidades com transtornos da aprendizagem, comunicação e déficits cognitivos, visando ao desenvolvimento de estratégias terapêuticas. Diante do panorama acima apresentado, o objetivo do presente trabalho é discutir a importância e as aplicações do exame neuropsicológico para o TDAH, utilizando como referência instrumentos disponíveis em nosso meio.

\section{Referências}

1. Kessler R, Adler L, Barkley R, Biederman J, Conners CK, Demler O, et al. The prevalence and correlates of adult ADHD in the United States: results from the national comorbidity survey replication. Am J Psychiatry. 2006;163:716-23.

2. Mattos P, Saboya E, Ayrão V, Segenreich D, Duchesne M, Coutinho G. Comorbid eating disorders in a Brazilian attention-deficit/hyperactivity disorder adult clinical sample. Rev Bras Psiquiatr. 2004;26:248-50.

3. Grevet E, Bau C, Salgado CA, Fischer AG, Kalil K, Victor MM, et al. Lack of gender effects on subtype outcomes in adults with attention-deficit/ hyperactivity disorder: support for the validity of subtypes. Eur Arch Psychiatry Clin Neurosci. 2006;5:1-9.

4. Coutinho G, Mattos P, Schmitz M, Fortes D, Borges M. Agreement rates between parents' and teachers' reports on ADHD symptomatology: findings from a Brazilian clinical sample. Rev Psiq Clín. 2009;36(3):101-4.

5. Dias G, Mattos P, Coutinho G, Segenreich D, Saboya E, Ayrão V. Agreement rates between parent and self-report on past ADHD symptoms in an adult clinical sample. J Att Dis. 2008;12(1):70-5.

6. Barkley R, Murphy K, Fischer M. ADHD in adults. New York: The Guilford Press; 2008.

7. Mattos P, Palmini A, Salgado C, Segenreich D, Grevet E, Oliveira I, et al. Painel brasileiro de especialistas sobre o diagnóstico do transtorno de déficit de atenção/hiperatividade (TDAH) em adultos. Rev Psiquiatr. 2006;28:50-60.

8. Barkley RA. Behavioral inhibition, sustained attention, and executive functions: constructing a unifying theory of ADHD. Psychol Bull. 1997;121:65-94. 
9. Willcutt EG, Doyle AE, Nigg JT, Faraone SV, Pennington BF. Validity of the executive function theory of attention-deficit/hyperactivity disorder: a meta-analytic review. Biol Psychiatry. 2005;57:1336-46.

10. Biederman J, Petty C, Fried R, Fontanella J, Doyle AE, Seidman LJ, et al. Impact of psychometrically defined deficits of executive functioning in adults with attention deficit hyperactivity disorder. Am J Psychiatry. 2006;163:1730-8.

11. Sonuga-Barke EJ. The dual pathway model of AD/HD: an elaboration of neuro-developmental characteristics. Neurosci Biobehav Rev. 2003;27(7):593-604.

12. Sagvolden T, Aase H, Zeiner P, Berger D. Altered reinforcement mechanisms in attention-deficit/hyperactivity disorder. Behav Brain Res. 1998:94:61-71.

13. Seargent J, Geurts H, Huijbregts S, Scheres A, Oosterlaan J. The top and the bottom of ADHD: a neuropsychological perspective. Neurosci Biobehav Rev. 2003;583-92.

14. Baddeley A. The episodic buffer: a new component of working memory. Trends Cogn Sci. 2000;4:417-23.

15. Baddeley A. Working memory: looking back and looking forward. Nat Rev Neurosci. 2003;4(10):829-39.

16. Coutinho G, Mattos P, Araújo C, Duchesne M. Transtorno do déficit de atenção e hiperatividade: contribuição diagnóstica de teste de atenção visual. Rev Psiq Clín. 2007;34(5):215-22.

17. Malloy-Diniz L, Leite W, De Moraes PH, Correa H, Bechara A, Duentes D. Brazilian Portuguese version of the Iowa Gambling Task: transcultural adaptation and discriminant validity. Rev Bras Psiquiatr. 2008;30:144-8.

18. Malloy-Diniz LF, Lasmar VA, Gazinelli LS, Fuentes D, Salgado JV. The Rey Auditory-Verbal Learning Test: applicability for the Brazilian elderly population. Rev Bras Psiquiatr. 2007;29(4):234-9.

19. McGough JJ, Barkley RA. Diagnostic controversies in adult attention deficit hyperactivity disorder. Am J Psychiatry. 2004;161:1948-56.
20. Gordon M, Barkley R. Tests and Observational Measures. In: Barkley RA, editor. Attention deficit hyperactivity disorder - a handbook for diagnosis and treatment. New York: The Guilford Press; 1999, p. 294-311.

21. Malloy-Diniz LF, Fuentes D, Borges W, Correa H, Bechara A. Impulsive behavior in adults with attention deficit/hyperactivity disorder: characterization of attentional, motor and cognitive impulsiveness. J Int Neuropsychol Soc. 2007;13:693-8.

22. Patton JH, Stanford MS, Barratt ES. Factor structure of the Barratt Impulsiveness Scale. J Clin Psychol. 1995;51:768-74.

23. Coutinho G, Mattos P, Malloy-Diniz LF. Neuropsychological differences between attention deficit hyperactivity disorder and control children and adolescents referred for academic impairment. Rev Bras Psiquiatr. 2009;31(2):141-4.

24. Amorim P. Mini International Neuropsychiatric Interview (MINI): validação de entrevista breve para diagnóstico de transtornos mentais. Rev Bras Psiquiatr. 2000;22:106-15.

25. Adaptação transcultural para o português da escala Adult Self-Report Scale para avaliação do transtorno de déficit de atenção/hiperatividade (TDAH) em adultos. Rev Psiq Clín. 2006;33(4):188-94.

26. Dias G, Mattos P, Coutinho G, Segenreich D, Saboya E, Ayrão V. Agreement rates between parent and self- report on past ADHD symptoms in an adult clinical sample. J Atten Disord. 2008;12:70-5.

27. Hervey AS, Epstein JN, Curry JE. Neuropsychology of adults with attention-deficit/hyperactivity disorder: a meta-analytic review. Neuropsychology. 2004;18:485-503.

28. Johnson DE, Epstein JN, Waid LR, Latham PK, Voronin KE, Anton RF Neuropsychological performance deficits in adults with attention-deficit/ hyperactivity disorder. Arch Clin Neuropsychol. 2001;16:587-604.

29. Biederman J, Petty C, Fried R, Black S, Faneuil A, Doyle A, et al. Discordance between psychometric testing and questionnaire-based deficitions of executive function deficits in individuals with ADHD. J Atten Disord. 2008;12:92-102. 